\title{
Selective exciton formation in thin GaAs-AlGaAs quantum wells
}

\author{
P.W.M. BLOM ${ }^{(1)}$, P.J. VAN HALL, C. SMIT, J.P. CUYPERS and J.H. WOLTER \\ Physics Dpt., Eindhoven University of Technology, P.O. Box, 5600 MB Eindhoven, The Netherlands
}

\begin{abstract}
We have found experimentally, that the exciton luminescence rise times in $\mathrm{GaAs} / \mathrm{AIGaAs}$ quantum wells oscillate as a function of incident laser energies. Guided by MonteCarlo simulations we interpret these results as the occurrence of selective LO-phonon assisted exciton formation.
\end{abstract}

In the photoexcitation of semiconductors excitons can be generated either directly with high momentum or by free electrons and holes [1]. In II-VI semiconductors the process of direct exciton formation is enhanced by the large exciton binding energy and by the strong electron-phonon coupling. The relaxation of these hot excitons gives rise to sharp resonances in the excitation and luminescence spectra [2]. In III-V semiconductors, where the exciton binding energy is much smaller, the photoexcited electrons and holes relax independently within their (sub)bands and subsequently form excitons by interaction with optical and acoustic phonons or by carrier-carrier scattering. So in this model the exciton dynamics are governed by carrier cooling, exciton formation and exciton relaxation and will be reflected in the risetime of the luminescence after laser excitation. The decay time is a measure for the exciton life time.

We have measured in a standard up-conversion experiment [3] this rise time for a narrow quantum well as a function of the laser energy. The sample consisted of 10 wells each $26 \mathrm{~A}$ wide. In such a well there is only one electron, h-hole and l-hole level. The results are given in fig. 1 and show a remarkable oscillating behaviour. From this we draw as a first conclusion, that the position in $\mathrm{k}$-space where the carriers land after the LO-phonon cascade might be of vital importance.

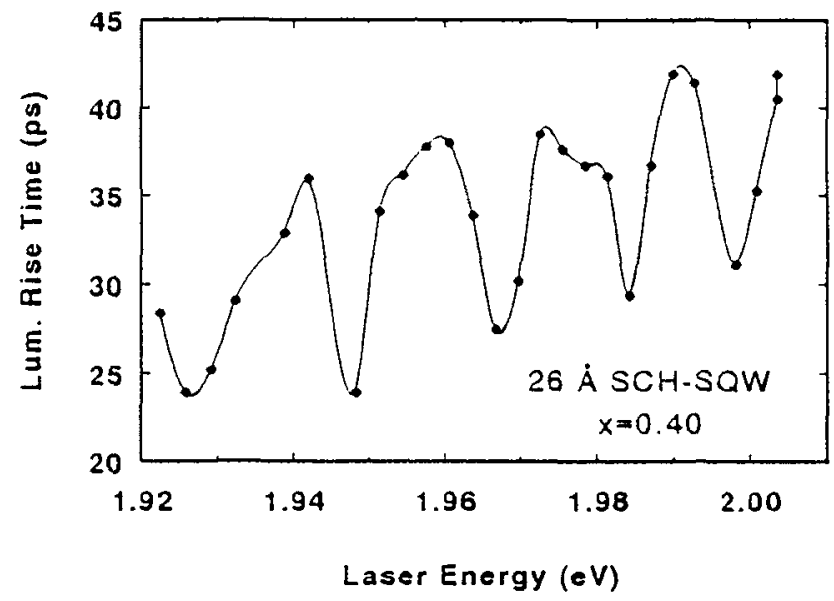

Fig.1 Experimental exciton luminescence rise times at $\mathrm{T}=8 \mathrm{~K}$ as a function of laser energy. Note the zero suppression of the scale.

(1) Present address: Philips Research Laboratories, Eindhoven, The Netherlands 
Such effects have already been searched for but with limited succes [4-6]. Only Roussignol et al. [6] have found a dependence of the rise time on the laser energy. Our experiment differs from those reported in the literature by the use of narrower well (26 A instead of $45 \mathrm{~A}$ or more) and of a laser energy well above ( about $250 \mathrm{meV}$ ) the exciton line. Also our excitation density is somewhat higher, which results in a nearly complete diffusion of the heavy-holes before the electrons have completed their LO-phonon cascade.

It might be useful to point out that a good ( $\mathrm{a}$ few meV) energy resolution of the laser beam is essential. This limits the time resolution to about 1 ps. For example a $100 \mathrm{fs}$ pulse, corresponding to an energy spread of about $20 \mathrm{meV}$, gives rise to a hot featureless distribution, with a complete loss of selectivity.

In order to find out whether such a concept of "sensitive spot(s)" makes sense in our experimental conditions, we have performed a series of Monte-Carlo simulations. Exchange effects in carrier-carrier scattering were accounted for by an effective enhancement factor [7]. The resulting time dependent luminescence data were processed as pseudo-experimental data and rise times were extracted.

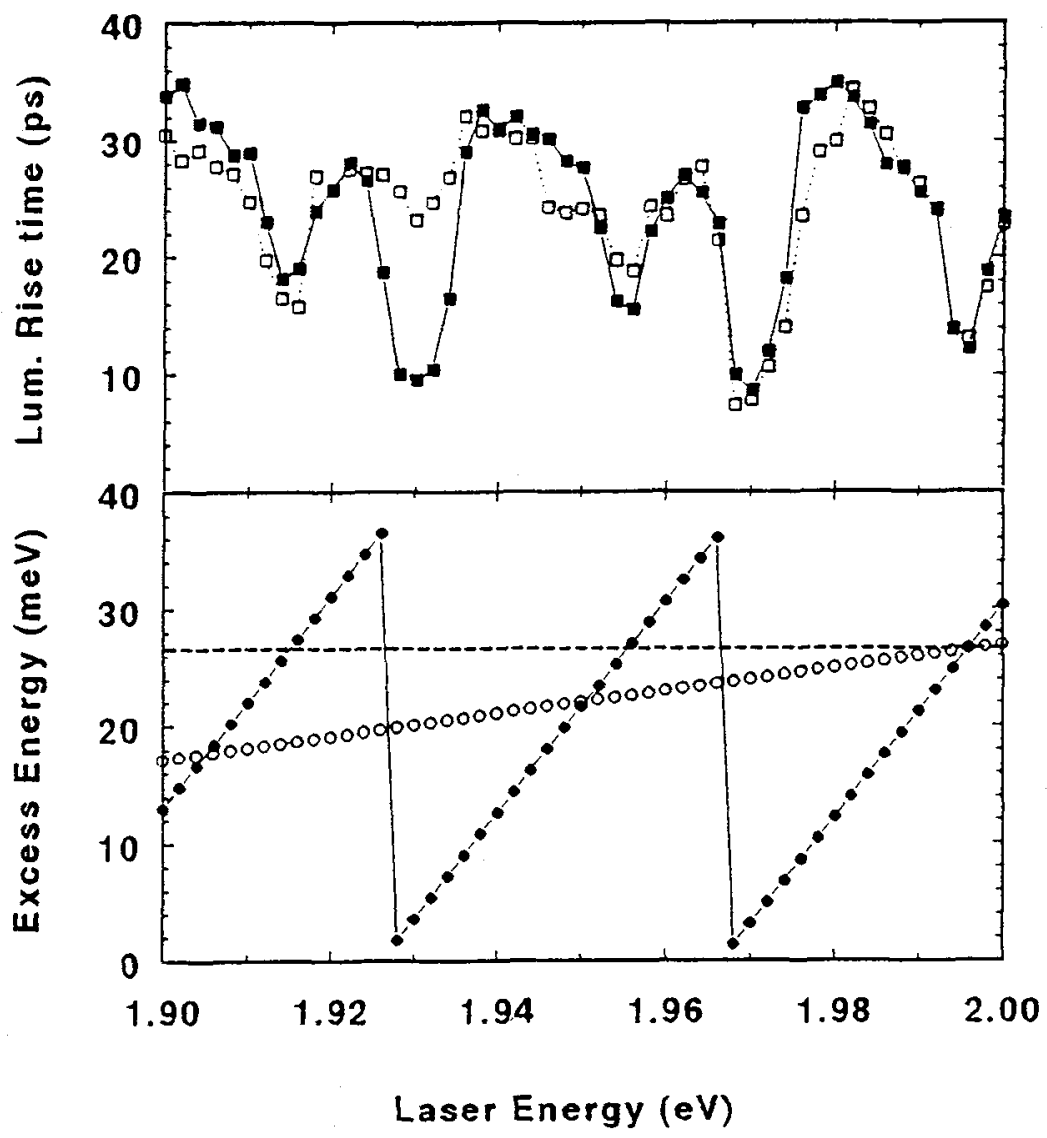

Fig.2a Calculated risetimes for $h$-hole excitation only $(\boldsymbol{E})$ and for the case where both $h$-hole and l-holes have been excited ( $\square$ ).

b Electron $(\bullet)$ and $h$-hole $(0)$ energies after the LO-phonon cascade. The dashed horizontal given the "resonance" condition of $26.6 \mathrm{meV}$. 
Since the electron-LOphonon coupling is also quit strong in III-V semiconductors we assumed LOphonon assisted exciton formation as the dominant channel. We employed three models for this process: $\mathrm{i}$ Whenever the sum of electron and $h$-hole energy was equal to the LO-phonon energy minus the exciton binding energy

$(10 \mathrm{meV})$ formation of an exciton in its ground state was possible. This model was not selective enough and produced a rather featureless structure. Only where accidentally all possible combinations of electron and hole energies satisfied the energy requirements an enhancement of the luminescence occurred. Obviously we need a further restriction in $\mathbf{k}$-space, as done in the other (ii and iii) possibilities.

ii In addition to the energy condition of $i$ we demanded a relative momentum $K \approx 0$.

This turned out to be too restrictive, giving rise more to retardation than to enhancement, viz. producing peaks instead of dips.

iii Here we used the additional condition, that one of the particles had $\mathbf{k} \approx 0$.

Some of the results using this model are shown in fig. 2 .

We clearly see a strong resemblance with the experimental results (fig.1). In the case where only electron-h-hole pairs have been excited the interpretation can be made very easily, using the lower part of the figure, where the energies after the LO-phonon cascade are depicted. Due to the large effective mass the heavy holes diffuse very fast by carrier-carrier scattering. So before the electrons have descended the LO-phonon ladder, $h$-holes with $\mathrm{k} \approx 0$ are present. When the electrons end with an energy of about $26 \mathrm{meV}$, all conditions are fulfilled and we have a fast rise of the luminescence (laser energies $1.915,1.955,1.995 \mathrm{eV}$ ). On the other hand the holes end their cascade with about $20 \mathrm{meV}$ over the whole range of laser energies. So due to the diffusion an appreciable amount will have an energy around $26 \mathrm{meV}$. Whenever the electrons end at $\mathrm{k} \approx 0$ the conditions are again fulfilled, albeit the other way around. This gives the sharper resonances at 1.930 and $1.970 \mathrm{eV}$. The fact that these dips are more pronounced is due to the fact that the diffusion of the holes is not infinitely fast, so there is still a bump around $20 \mathrm{meV}$, when the electrons have completed the LO-phonon cascade.

If we also allow for light-hole excitation, some of the features are washed out a bit. This can be explained in a similar way as the discussion given above, taking into account the other excess energies of electrons and holes, the latter after a th-hh interband transition.

It should be emphasized, that in these calculations we used simple parabolic bands with the bulk effective masses. So the results can be qualitative only. A detailed comparison between experiment and theory requires a precise knowledge of the band structure, which is hard to obtain especially for the hole bands. Theoretical calculations [8] indeed have shown the complexity for the sample used in our experiments. Using the results of this calculation we have obtained a reasonable agreement between the calculated rise times and the results given in fig.1 [9]

We have looked experimentally for a possible preference of exciton formation in which one of the particles is at rest at the bottom of the band viz. our model (iii). For this we used a similar sample, but now n-doped. This supplies us with a large amount of electrons with $k \approx 0$. Indeed we found at some laser energies an additional enhancement of the exciton luminescence, though the risetimes being larger by a factor of about two (see fig.3). This is a clear evidence for the special role of the point $\mathrm{k}=0$. A similar experiment with p-type material did not show such effects. Obviously the fast hole-hole scattering supplies us with sufficient heavy holes at $k \approx 0$.

In conclusion we have observed oscillations in the exciton luminescence rise times. The nature of these resonance-like oscillations arises from LO-phonon assisted exciton formation. Monte-Carlo simulations suggest strongly that one of the paticipating particles should have zero energy. Moreover a theoretical derivation of the transition probability of LO-phonon assisted exciton formation [8] indeedgives a maximum probability for the situation, where the h-hole has zero energy. This idea is corroborated by experiments with doped samples, giving a consistent picture of this phenomenon. 


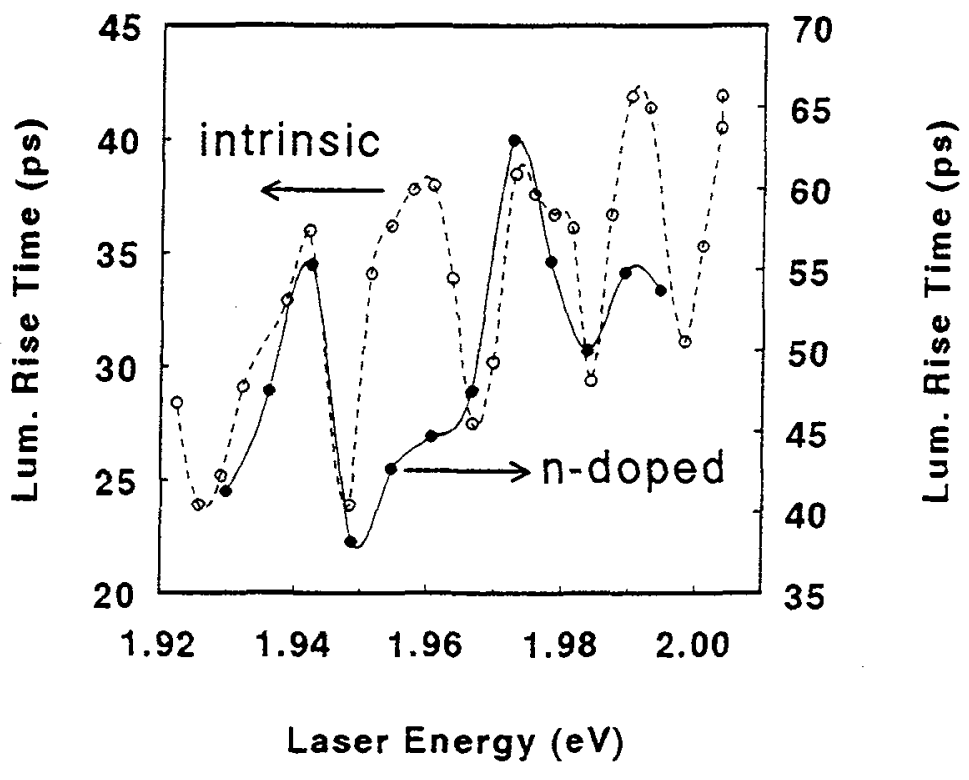

Fig.3 Comparison between exciton luminescence rise times for an undoped ( 0 ) and an n-doped ( $\bullet$ ) sample. Note the change in scale.

\section{References}

[1] Permogorov S., Phys. Stat. Sol. (b) 68 (1975) 9.

[2] Stanley R.P., Hegarty J., Fischer R., Feldmann J., Gobel E.O., Feldmann R.D. and Austin R.F., Phys. Rev. Lett. 67 (1991) 128.

[3] Blom P.W.M., Thesis Eindhoven University (1992).

[4] Damen T.C., Shah J., Oberli D.Y., Chemla D.S., Cunningham J.E. and Kuo J.M. Phys. Rev. B42 (1990) 7434

[5] Deveaud B., Clerot F., Roy N., Satzke K., Sermage B. and Katzer D.S. Phys. Rev. Lett. 67 (1991) 2355

[6] Roussignol Ph., Delalande C., Vinattierri A., Carraresi L. and Colocci M. Phys. Rev. B45 (1992) 6965

[7] Van Hall P.J. and Blom P.W.M., Superl. and Microstr. 13 (1993) 329

[8] Cuypers J.P, and Van Haeringen W, Condens. Matter 4 (1992) 2587.

[9] Blom P.W.M. and Van Hall P.J. to be published 